\title{
Theoretical Research on the Import and Export of Iron Ore and Harmful Element Detection Method based on Microwave Digestion Inductively Coupled Plasma Mass Spectrometry Approach
}

\author{
$\mathrm{Nan} \mathrm{Wu}^{1}$ \\ ${ }^{1}$ Hebei entry-exit inspection and Quarantine Bureau Office of Caofeidian , \\ Tangshan,Hebei,China
}

\begin{abstract}
In this paper, we conduct research on the import and export of iron ore and harmful element detection method based on microwave digestion inductively coupled plasma mass spectrometry approach. Our country gradually increased demand for iron ore, iron ore trade, in order to ensure that the quality of the iron ore and taste which must be tested for total iron content. At the same time it also increased the demand for iron ore, although China's iron ore production has been greatly improved. Our proposed approach optimizes the traditional detection method which will be meaningful.
\end{abstract}

Keywords: Harmful Element Detection; Microwave Digestion; ICP-MS; Import and Export.

\section{Introduction}

Iron ore demand in China is one of the biggest imports, imports of the first in the world. Import countries including Australia, India, Brazil, the main producing countries such as Iran, Venezuela. As a kind of mineral resources, iron ore for the development of the national economy to bring vitality and vigor at the same time, also brought heavy metal pollution and its associated. Along with our country economy growth, study on the mining area environment safety in China is more and more attention, for is closely related to people's life in the soil, drainage standard for controlling poisonous and harmful elements such as perfecting just, but of mass immigration and mobility strong poisonous and harmful elements in iron ore and the impact of environment and the health of our country not to carry out the system research. Due to the rapid economic development of our country, the domestic market demand for steel, increasing steel production in China. Our country gradually increased demand for iron ore, iron ore trade, in order to ensure that the quality of the iron ore and taste, must be tested for total iron content, current detection methods used have instrument testing and chemical testing, the testing technology have their own characteristics and advantages, has been widely used in the iron and steel industry. At the same time it also increased the demand for iron ore, although China's iron ore production has been greatly improved. As the main importer of iron ore in the detection of poisonous and harmful elements in the system of census and the elements of soil and water system is evaluated and the influence of finding out the main influence factors which puts forward the requirements.

Industry of our country has developed rapidly in recent years, iron ore testing frequency is becoming more and more high, at the same time, also exposed some problems: the first is testing workload is bigger, in general, many operations such as drying and so on all need to manually professional testing personnel, rapid increase in the number of test, test workload has increased, testing personnel number is difficult to meet demand. The second is the detection cycle is too long, the increased number is bound to cause delay detection work, coupled with the inspectors 
may occur in the process of detecting errors or negligence, detection quality problem, also extend the detection cycle. The third is the resource consumption is too big, we need to use a lot of power, water and other chemicals can complete the ore testing, the quantity of detection also brought resources consumption in great quantities and a large number of chemicals can also cause environmental pollution problems.

When testing for iron ore concentrate, natural such as iron ore is suitable for chemical test, mainly used in the chemical reaction, first of all, fusing or acidification, or use a strong acid, strong alkali dissolved the iron ore samples, using titanium or stannous chloride to high iron reduction for low iron ion, and then use the potassium dichromate to excess reductant to oxidation, using diphenylamine sulfonate as an indicator, join the potassium dichromate titration solution, arrived at the titration end point calculations using a total amount of potassium dichromate solution, according to the relevant parameters are calculated total iron content of iron ore. In general, because of the low concentration of rare earth elements in the samples, the chemical properties is very similar, before the determination of the need for separation and enrichment, the complicated analysis process, the above analysis methods are subject to different degrees of matrix interference and coexisting element interference and in some cases, its application is seriously restricted [1-3].

In this paper, we conduct research on the import and export of iron ore and harmful element detection method based on microwave digestion inductively coupled plasma mass spectrometry approach. Rare earth element geochemical tracer is good, the geochemical characteristics of the study on mineralogy, ore deposit study fields such as rock formation has been widely used. Accurate determination of trace rare earth elements in geological samples quickly geochemical analysis and test technology has been the research focus in the field. Iron ore is rich in rare earth elements, along with the industrialization process in our country, iron ore imports of progressing for accurate determination of rare earth elements in iron ore is one of the main research topic in the field of the current mineral detection. In the following sections, we will discuss the issues in detail.

\section{Our Proposed Methodology}

The Basic Introduction of Iron Ore and Harmful Element Detection. Imports of iron ore mainly through dust and stored near the rains washed out into the water. Poisonous and harmful elements, especially mercury, cadmium, lead, chromium has significant biological toxicity. They cannot be microbial degradation in water and can only happen on various forms of mutual transformation and dispersion and concentration process. The pollution characteristic is: besides was taken away by suspended solids, due to precipitation and adsorption concentration in sediment and become long-term secondary pollution. Water of the various organic and inorganic ligand coordination experience rather than generate complex or chelate which will cause the poisonous and harmful elements have greater water solubility and has entered the poisonous and harmful elements of sediment and may be released again. Being able to bring people to the water body of iron ore very few, the poisonous and harmful elements has little influence for surface water, and immersed in water after the rain washing ore or its solute the environmental impact of further research. Ore particles because floats, wind and other dust into the atmosphere will cause harm to surrounding environment with, especially for those nearby, mainly by people of breathing and directly into the body, may also enter the human body through contact or irritate the skin. Iron ore dust harm to human body depends on its chemical composition and concentration. Free silica content in iron ore is very low, there is no harm to human body, mainly is poisonous and harmful elements enter the body 
through breathing and produce certain effect which will cause serious result and influence.

Table 1.The Standard Trace Element Numerical Data

\begin{tabular}{ccccccc}
\hline $\mathrm{Cd}$ & $\mathrm{Hg}$ & $\mathrm{As}$ & $\mathrm{Pb}$ & $\mathrm{Cu}$ & $\mathrm{Zn}$ & $\mathrm{Ni}$ \\
\hline 0.10 & 0.05 & 10.0 & 20.0 & 30.0 & 80.0 & 35 \\
\hline 0.15 & 0.25 & 15.0 & 30.0 & 41 & 100 & 45 \\
\hline 0.84 & 0.25 & 16.0 & 350 & 265 & 700 & 67 \\
\hline 1.3 & 0.35 & 19.0 & 500 & 375 & 1000 & 95 \\
\hline
\end{tabular}

Imported iron ore shipped from former mainly stacked in the port area and the surrounding freight yard, mainly affect the region's soil, because no direct contact with the surrounding farmland, so here only discussion of poisonous and harmful elements in the iron ore to freight yard area the potential impact of soil. Inductively coupled plasma emission spectrometry with high sensitivity, rapid detection of multiple elements at the same time, the existing literature on determination of impurity elements in manganese ore by microwave digestion inductively coupled plasma mass spectrometry method, but the sample pretreatment wet or alkali fusion method is used to dissolve the sample, process complex detect interference is serious. Have been reported with hydrofluoric acid and mixed acid, microwave digestion sample after being dissolved sample, in order to reduce the hydrofluoric acid damage to the instrument and the introduction of the boric acid masking fluorine ions, which not only increased the workload, at the same time the introduction of boric acid and brings to the determination of the interference. Therefore, analyzing the components of the iron is urgently needed.

The Set-up of the Experiment. The instrument we plan to use is listed as the follows. (1) ICAP6300 full spectrum direct reading plasma emission spectrometer. (2) Contain Ceramic Centre tube, HF Resistant Nebuliser and HF Resistant Spray Chamber with Spray Chamber Adaptor. (3) IRIS Advantage full spectrum direct reading plasma emission spectrometer. (4) Agilent 7500 a type inductively coupled plasma mass spectrometer (Agilent companies in the United States), equipped with Barbinton atomizer. The operation parameters are shown in the table two.

Table 2.The Operation Parameters for the Proposed Experiment

\begin{tabular}{|c|c|c|c|}
\hline Parameter & Condition & Parameter & Condition \\
\hline RF Power & $1200 \mathrm{~W}$ & $\begin{array}{l}\text { Sampling } \\
\text { Depth }\end{array}$ & $7.5 \mathrm{~mm}$ \\
\hline $\begin{array}{c}\text { Carrier Gas } \\
\text { Flow Rate }\end{array}$ & $1.1 \mathrm{~L} / \mathrm{min}$ & $\begin{array}{l}\text { Integral } \\
\text { Interval }\end{array}$ & $0.1 \mathrm{~s}$ \\
\hline $\begin{array}{l}\text { Atomizing } \\
\text { Speed }\end{array}$ & $0.1 \mathrm{r} / \mathrm{s}$ & $\begin{array}{l}\text { Repeat the } \\
\text { Number }\end{array}$ & $2 \sim 3$ \\
\hline $\begin{array}{l}\text { Sample } \\
\text { Cone }\end{array}$ & $\mathrm{Ni}$ & $\begin{array}{c}\text { The } \\
\text { Sensitivity }\end{array}$ & $1000 \mathrm{cps}$ \\
\hline
\end{tabular}

Standard and the main reagent could be summarized as the follows. (1) More than $10 \%$ HNO, medium element mixed standard stock solution (8500-6940). Cd, Cr, As, and $\mathrm{Pb}$ concentrations of $10 \mathrm{mg} / \mathrm{L}$. (2) $2 \% \mathrm{HNO}$, tuned liquid medium mass spectrometry: $\mathrm{Li}, \mathrm{Y}, \mathrm{Ce}, \mathrm{Ti}$, Co concentration is $10 \mathrm{~g} / \mathrm{L}$. (3) $10 \% \mathrm{HNO}$, medium single element $\mathrm{Hg}$ standard stock solution (8500-6941), the concentration of 10 $\mathrm{mg} / \mathrm{L}$. (4) Ultrapure water, in line with $\mathrm{GB} / \mathrm{T}$ 6682 .

The Sample digestion program design is shown in the table three. Quick update simplifies the analysis steps of analysis instrument, improve the efficiency. If for iron ore and the concentrate on the determination of elements in the conventional pretreatment methods, there must be due to the high salt concentration effect the precision of instrument, even damage instrument parts, etc. Microwave digestion technology is a combination of high pressure airtight digestion and microwave rapid heating performance and both has the sample dissolved completely, fast, low blank, less reagent consumption and avoid volatile elements loss and high recovery rate. Accurately and timely can not only meet the needs of production and scientific research, make up for the determination of trace elements in wet accuracy and detection limit, the deficiency of the applied to the fixed value of standard sample 
analysis at the same time, compared with atomic absorption and chemical analysis method, has the short analysis period, high accuracy, the characteristics of low detection limit, has great practical value. Coexistence elements produce line may cause spectrum interference, so when choosing analytical spectral lines which usually choose low background, letter back than high, high sensitivity, treat measurement matrix element spectral interference or interference is very small.

Table 3.The Sample Digestion Program Design

\begin{tabular}{cccc}
\hline $\begin{array}{c}\text { Digestion } \\
\text { Steps }\end{array}$ & Temperature & Time & $\begin{array}{c}\text { Time } \\
\text { Kept }\end{array}$ \\
\hline 1 & $152 \sim 161$ & $2 \sim 6$ & $3 \sim 4$ \\
2 & $170 \sim 190$ & $2 \sim 6$ & $10 \sim 15$ \\
3 & 95 & 2 & 5 \\
4 & 95 & 2 & 0 \\
\hline
\end{tabular}

The Experimental Result Analysis. Microwave digestion is fast digestion, sample digestion completely, less pollution, high recovery rate, etc. In recent years is widely used in the pretreatment of the sample. Due to the elements in the sample under the excitation of the high frequency plasma, produce thousands of spectral lines, thus to analyze certain elements, must first to select line. Generally, the preferred instrument sensitive recommended by the first line; In the other elements to analysis element line does not produce interference, choose the background as far as possible small concentration, low excitation level line. Experimental results show that with the change of the acidity of the solution of the measured value changes, with $5 \%, 15 \%, 20 \%$ of hydrochloric acid concentration measurement value of each element has a great change, unstable when the solution of hydrochloric acid concentration of $10 \%$ each element basic conform to the standard measurements. This suggests that the hydrochloric acid when the concentration of $10 \%$ to minimal impacts on the result of the measurement, so the experiment selected a solution of hydrochloric acid concentration of $10 \%$. Using inductively coupled plasma emission spectrometry genesis of multiple elements, the method of rapid detection of molybdenum has a wide linear range, convenient, and less reagent dosage, at the same time, this method greatly improves the work efficiency, reduce the energy consumption and has a good application prospect. The figure one illustrates the corresponding result. 


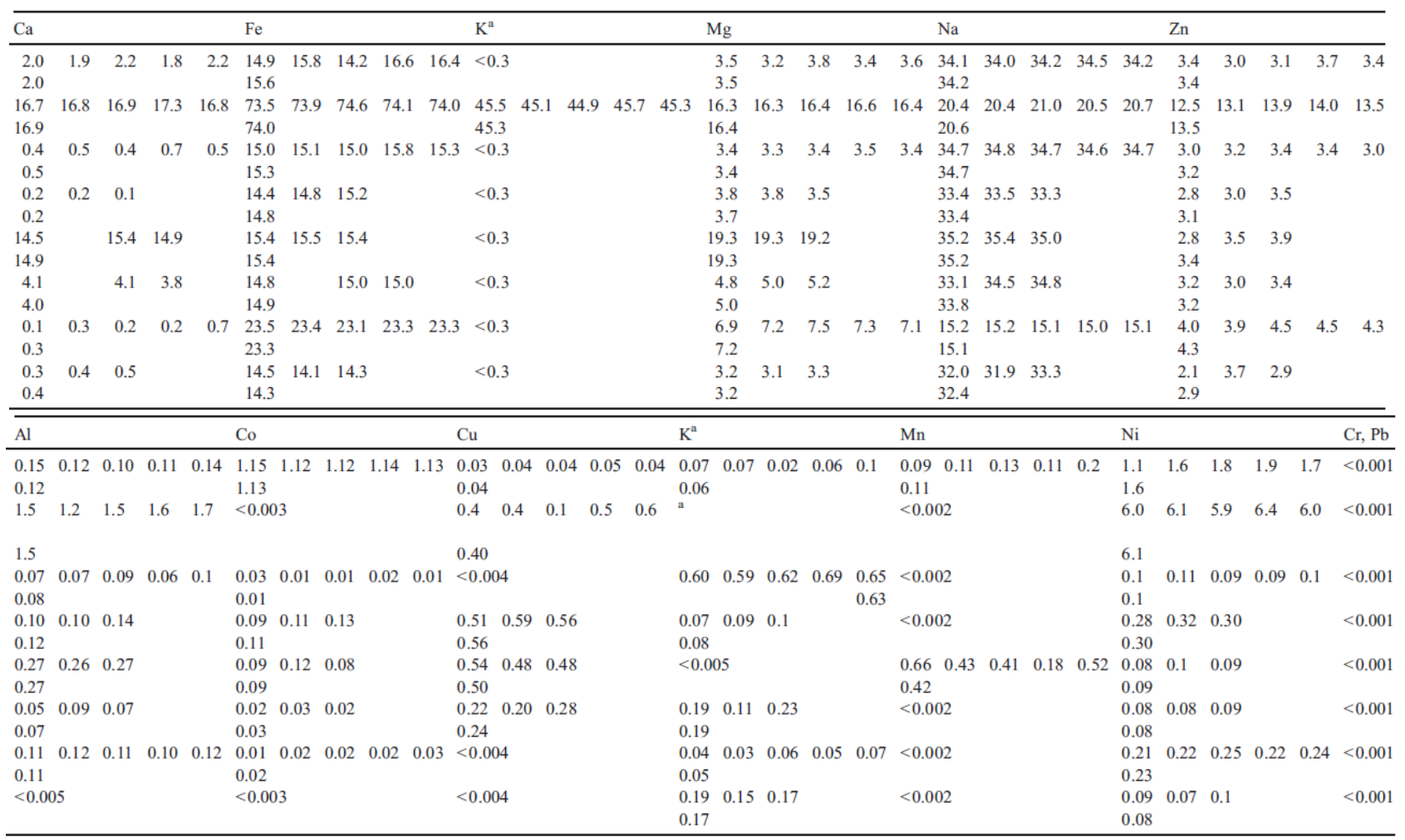

Figure 1.The Experimental Result for the Proposed Methodology

\section{Conclusion}

In this paper, we conduct research on the import and export of iron ore and harmful element detection method based on microwave digestion inductively coupled plasma mass spectrometry approach. Optimizing the microwave digestion and ICP ion source and the parameter of the mass spectrometer, and the standard addition method combining elements of interference correction equation, the complex ore to eliminate matrix and coexist elements, and conducted experiments such as precision, detection limit, accuracy, and the result is good and satisfactory. In the future, we have scheduled to conduct more literature review to optimize and modify the methodology.

\section{References}

[1] Jensen, S. K., Lacaze, J. P., Hermann, G., Kershaw, J., Brownlow, A., \& Turner, A., et al. (2015). Detection and effects of harmful algal toxins in scottish harbour seals and potential links to population decline. Toxicon, 97, 1-14.

[2] Qing-Bo Y I, Zheng Y Y, Liang F, et al. The Dynamic Simulation of Finite Element Method and Analysis on Detection Data for Tunnel Excavation and Supporting[J]. Guizhou Science, 2014.

[3] Johnsen S F, Taylor Z A, Han L, et al. Detection and modelling of contacts in explicit finite-element simulation of soft tissue biomechanics.[J]. International Journal of Computer

Assisted. 